\section{Synchronous occurrence of odontogenic keratocyst and ameloblastoma: A case report and review of the literature}

\author{
Norman Firth, ${ }^{1}$ Abdulhameed Alsarraf, ${ }^{2}$ \\ Nathan Vujcich, ${ }^{3}$ Omar Kujan ${ }^{4}$ \\ ${ }^{1}$ School of Dentistry, University of \\ Queensland, Herston QLD, Australia; \\ ${ }^{2}$ Oral Medicine clinics, Ministry of \\ Health, Kuwait; ${ }^{3}$ Department of Oral \\ and Maxillofacial Surgery, Royal Perth \\ Hospital, Perth WA, Australia; ${ }^{4}$ UWA \\ Dental School, University of Western \\ Australia, Nedlands WA, Australia
}

\begin{abstract}
Odontogenic keratocyst (OKC) and ameloblastomas are distinct histopathologically diagnosed odontogenic lesions of the oral cavity. Both are primarily located in the posterior regions of the mandible, however, they can involve the maxilla as well. The occurrence of both an OKC and ameloblastoma in a patient is very uncommon. This case demonstrated such a lesion in the mandible of a 57 years old female. The diagnostic work-up and features of both lesions are illustrated with special focus on histopathological variances distinguishing OKC from ameloblastoma with the support of immunohistochemistry. This case highlights the importance of identifying accurate diagnoses for such lesions which may prompt clinical implications. Clinico-pathologic understanding of both lesions signifies the need for careful management plan and prevention of recurrence. Previously reported simultaneous occurrences of odontogenic cysts and/or tumors in the oral cavity are also reviewed.
\end{abstract}

\section{Introduction}

Odontogenic keratocyst (OKC) is a developmental cyst of the oral cavity which was previously termed keratocystic odontogenic tumor (KCOT). ${ }^{1}$ The latest edition by the World Health Organization (WHO) for the classification of Head and Neck Tumors 2017 defined OKC as a cyst of odontogenic origin, characterized by a thin, regular lining of parakeratinized stratified squamous epithelium with palisading hyperchromatic basal cells. ${ }^{1}$ OKC may present as solitary or multiple arising from remnants of the dental lamina. Where multiple presentations occur, it is usually one of the stigmata of the inher- ited nevoid basal cell carcinoma syndrome. ${ }^{1}$ These cases tend to occur in younger age group patients. There is evidence of the association of PTCH gene in the etiology of OKCs in the context of mutation or inactivation of the PTCH1 gene. ${ }^{2}$ This in turn, activates the sonic hedgehog signaling pathway resulting in aberrant cell proliferation of OKC epithelium. ${ }^{2}$ The occurrence of OKCs was found to be from the first to the ninth decades of life, with a peak incidence is however in the second and third decades. ${ }^{3}$ The mandible is mostly affected with the angle of the mandible being a very common anatomical site. Another feature of OKCs is their high recurrence rate. ${ }^{4}$

Ameloblastoma is a benign epithelial odontogenic tumor characterized by expansion and a tendency for local recurrence if not adequately removed. ${ }^{1}$ It exhibits a progressive growth pattern and a locally invasive behavior with various histologic variants..$^{5}$ That said, the solid/multicystic is the most common comprising $91 \%$ of all ameloblastomas. ${ }^{6}$ Other histologic variants include the desmoplastic type, unicystic type and the extraosseous/peripheral type. ${ }^{1}$ Ameloblastomas most commonly affect the mandible but can involve the maxilla. The global incidence has been estimated at 0.5 cases per million person years, and diagnosis is made mostly in patients 30-60 years of age. ${ }^{6}$ Similar to the previously mentioned feature of $\mathrm{OKC}$, ameloblastomas has a welldocumented propensity for loco-regional invasion and risk of recurrence. ${ }^{5}$

The present case describes the synchronous occurrence of $\mathrm{OKC}$ and ameloblastoma in the mandible of a 57 years old female. Clinico-pathologic understanding of both lesions signifies the need for careful management plan and prevention of recurrence. The reported case was prepared in accordance with Helsinki Declaration and the patient provided an informed consent to publish this case.

\section{Case Report}

A 57 years old female was referred for private Oral and Maxillofacial Surgery consultation for evaluation and management of a two centimeters unilocular lesion between teeth 42 and 43 . The lesion was asymptomatic and therefore the duration of its presence was unknown. There was no alveolar swelling, no periodontal probing defects associated with the associated teeth 42 to 44. The 42 and 43 were cold responsive, suggesting pulpal integrity. The patient had presented with an orthopantomogram (OPG) which showed a $15 \mathrm{~mm}$ unilocular lesion located between the 42 and 43 roots,
Correspondence: Omar Kujan, UWA Dental School, University of Western Australia, 17 Monash Avenue, Nedlands WA 6009, Australia. Tel.: +61.8.64577649.

E-mail: omar.kujan@uwa.edu.au

Key words: Synchronous; odontogenic; ameloblastoma; keratocyst.

Contributions: NF and OK conceived the presented case, acquisition and collection of the data, analysis and critical interpretation of the data, and revising the manuscript. AA drafted the manuscript, and analyzed the literature. NV provided the treatment and followed up the case. All authors contributed to the revision of manuscript and approved the final version.

Conflict of interest: the authors declare no potential conflict of interest.

Ethics approval: The reported case was prepared in accordance with Helsinki Declaration.

Informed consent: Patient has provided informed consent.

Received for publication: 15 September 2019. Revision received: 23 May 2020.

Accepted for publication: 17 June 2020 .

This work is licensed under a Creative Commons Attribution NonCommercial 4.0 License (CC BY-NC 4.0).

(C) Copyright: the Author(s), 2020

Licensee PAGEPress, Italy

Clinics and Practice 2020; 10:1205

doi:10.4081/cp.2020.1205

which were displaced but not resorbed (Figure 1A). It extended apically only to the root of the canine, and was distal to the mental foramen. Non-contrast low-dose multidetector computed tomography (CT) of the mandible was then performed. This demonstrated a $15 \times 10 \times 8 \mathrm{~mm}$ unilocular expansile lesion with a corticated margin causing divergence of the roots of 42 and 43 without root resorption (Figure 1B). In addition, the labial cortex was thinned and elevated with a $5 \mathrm{~mm}$ perforation.

Clinical and radiological findings were suggestive of an OKC and the patient was consented to surgical treatment. Following surgical management of the lesion, subsequent histopathological and immunohistochemical examinations were performed. This demonstrated characteristic features of both OKC and ameloblastoma.

The management of the presented case aimed to surgically resect the lesion with the target of preventing recurrence by surgical enucleation of the lesion under general anesthetic as a presumptive OKC. 
Following enucleation, bony ostectomy was performed of the lower aspect of the cavity and Carnoy's solution was then applied for 3 minutes before irrigation and closure of the wound. Multiple follow-ups were performed. To date, the follow-up of more than one-year duration was insignificant with regards to the risk of recurrence or other complications.

Histopathological analysis of hematoxylin and eosin stained slides shown in Figure 2A and $\mathrm{B}$ demonstrates characteristic features of both OKC and ameloblastoma. The section in Figure 2A shows a cyst with a thick fibrous connective tissue wall, lined by parakeratinized stratified squamous epithelium with a columnar basal layer. For the most part, the epithelium does not have rete ridges, and is even in thickness, however, in some regions, rete ridge formation is present. Overall, this cyst has the morphology of an OKC. Several satellite cysts are present, and in regions of the fibrous connective tissue wall, clusters of epithelial islands with a columnar basal layer with peripheral palisading and reverse polarity and stellate reticulum-like material within the islands. A sparse chronic inflammatory infiltrate is present in the connective tissue wall in some areas.

The basic histopathologic patterns of ameloblastoma are demonstrated in Figure 2B. The follicular pattern is demonstrated with islands of odontogenic epithelium within a fibrous stroma in which the cells are palisaded, columnar and hyperchromatic. Some features of the plexiform pattern can also be seen with basal cells arranged in anastomosing strands and a stroma with cyst-like degeneration. Figure $2 \mathrm{C}$ shows a section involving both $\mathrm{OKC}$ and ameloblastoma. A panel of biomarkers (Ki-67, calretinin, CD56, and pan cytokeratin AE1/AE3) were immunohistochemically stained to confirm the diagnosis of ameloblastoma. OKC showed a high proliferative index demonstrated by the high Ki-67 staining whereas ameloblastoma epithelial island showed no such activity (Figure 3A). Calretinin was negative in both $\mathrm{OKC}$ and ameloblastoma contrary to the positive staining of pan cytokeratin in both lesions. Interestingly, CD56 (NCAM) was positively stained only in ameloblastoma tumor islands (Figure 3B).

\section{Discussion and Conclusions}

We hereof illustrated the synchronous occurrence of OKC and ameloblastoma in the mandible of a 57 years old female. An insight to the clinico-pathologic correlations of both pathological entities is impor- tant due to the high recurrence rates and the need for post-surgical follow-up. It is noteworthy to refer to the challenging nature of clinically diagnosing $\mathrm{OKC}$ with the use of radiographic imaging techniques and subtle clinical history. Recent literature has begun to enhance the use of imaging to distinguish between OKC and ameloblastoma. ${ }^{7}$ Generally, OKC is classified as a pathological entity which was previously termed KCOT by the WHO for the classification of Head and Neck Tumors in 2005 attributed to their neoplastic nature. ${ }^{1}$ The genetic profile of $\mathrm{OKC}$ involves genetic alterations in the PTCH1 gene where loss of heterozygosity has been found on the $9 \mathrm{q} 22.3$ region in OKCs and other developmental cysts. ${ }^{2}$ Despite the evidence of the associated genetic profile, further research is necessary to support the neoplastic nature of $\mathrm{OKC}$ and therefore it has been suggested that $\mathrm{OKC}$ remains the most suitable term for this lesion. ${ }^{1}$ On radiographic assessment, unilocular or multilocular radiolucencies

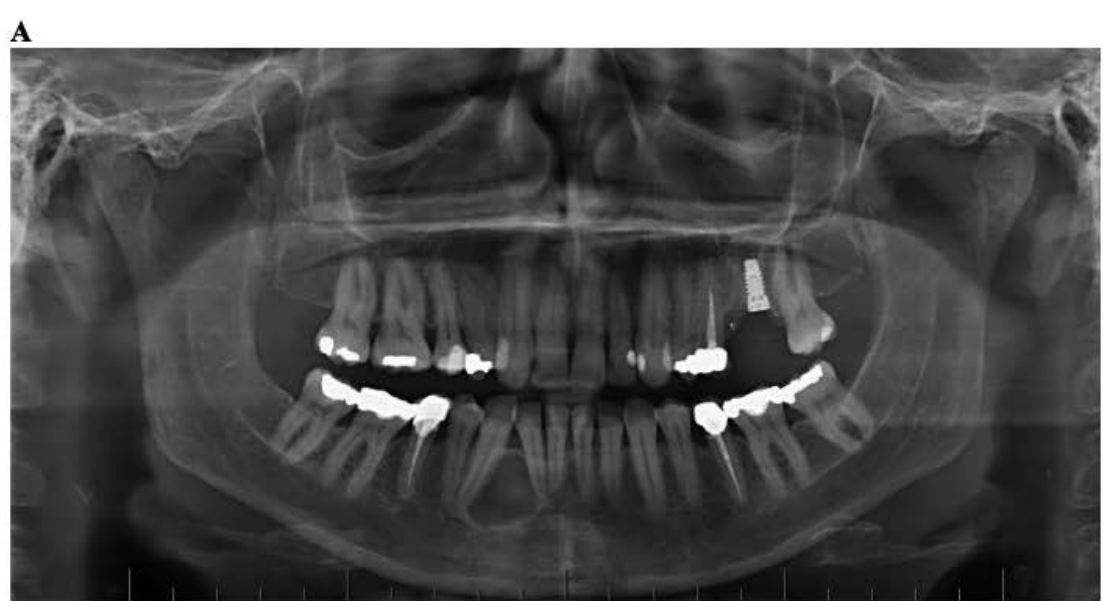

B

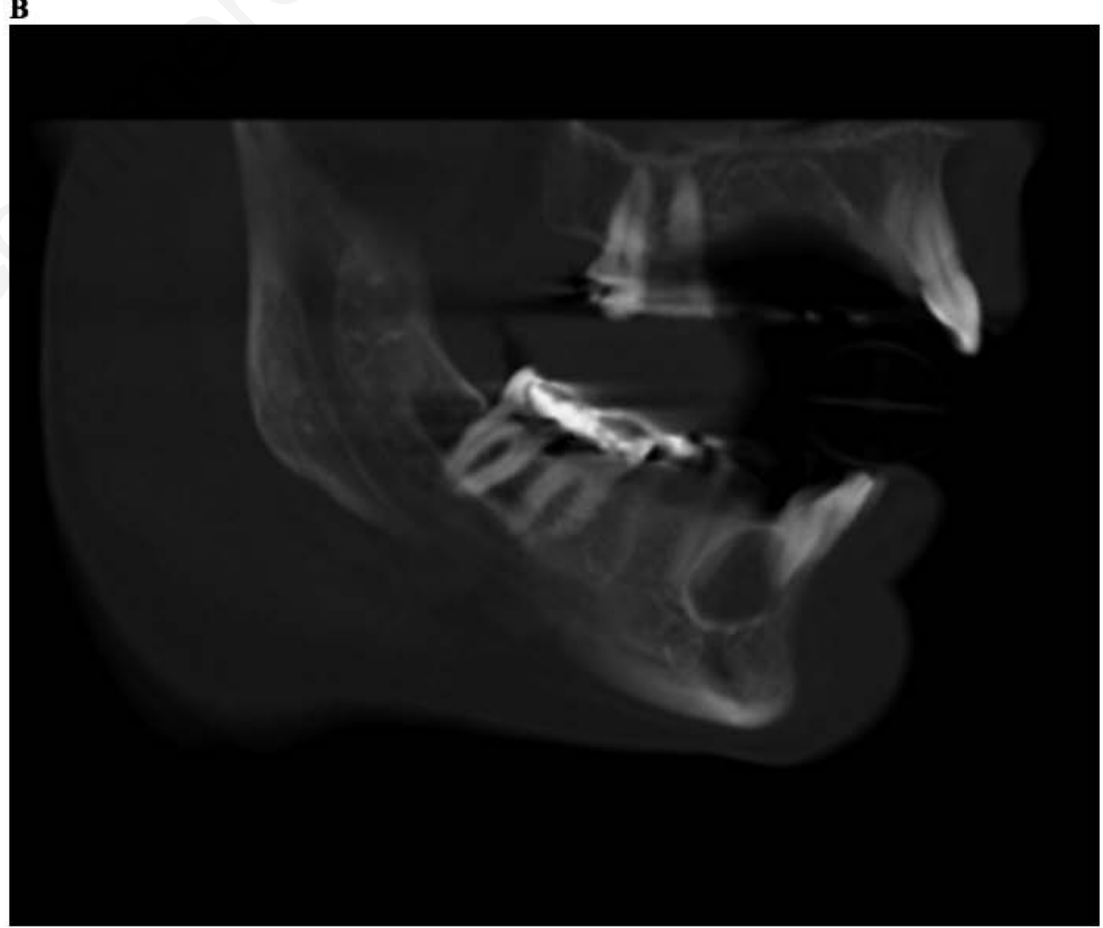

Figure 1. A) An orthopantomogram showing a $15 \mathrm{~mm}$ unilocular lesion located between the 42 and 43 roots, which were displaced but not resorbed. B) Multidetector computed tomographic mandibular Dentascan (axial view). Demonstration of root divergency of teeth 42 and 43 . In the right anterior mandible, the $15 \mathrm{~mm}$ diameter uniloculate expansile low-density lesions which diverges the intact roots of 42 and 43 was suggestive of odontogenic keratocyst. 
with thin, reactive and sclerotic bony borders and smooth or scalloped margins can be seen. ${ }^{7}$ In some occasions, locally destructive lesions may demonstrate invasion into bone radiographically. ${ }^{7}$

On the other hand, ameloblastoma is a benign, progressively-growing and locally invasive odontogenic tumor. Ameloblastoma originates from remnants of the dental lamina, indicated by the expression of early dental epithelial markers such as PITX2, MSX2, DLX2, RUNX1 and ISL $1 .{ }^{8}$ The clinical presentations vary from incidental findings on imaging to painless swellings and pain or rapid growth in invasive ameloblastomas. ${ }^{9}$ In desmoplastic ameloblastomas, tooth displacement and root resorption may be seen, although infrequent. ${ }^{10}$ The diagnostic workup for the patients with ameloblastoma, regardless of the type, include imaging and surgical biopsy. The former includes $\mathrm{CT}$ as the most useful imaging modality which can demonstrate well-defined unilocular or multilocular radiolucencies and expansile lesions. ${ }^{9}$ In addition, magnetic resonance imaging (MRI) is helpful in demonstrating soft tissue and marrow extension beyond the lytic bone cavity and help characterize extension to the orbit, paranasal sinuses and skull base when ameloblastomas arises in the maxilla. ${ }^{11}$ A biopsy on the other hand, provide tissue for histopathological assessment which establishing a definitive diagnosis. It may also be useful to avoid unnecessary operations for differential diagnoses such as $\mathrm{OKC}$, aneurysmal bone cysts, or central giant cell lesions. ${ }^{5}$ The current standard care for ameloblastomas can be contentious, mainly determined by the histopathological subtype (non-mural unicystic forms versus solid multicystic types). Some authors may still advocate less aggressive treatment with enucleation/curettage. ${ }^{12}$ However, radical surgery with 1-centimeter bone margins is generally advocated for solid multicystic forms and intramural unicystic forms. This may create segmental defects, or at least involve a marginal mandibulectomy or oro-antral communication in the maxilla. ${ }^{13,14}$ There are several factors that affect the prognosis of ameloblastoma management. These include patient's age, tumor size, extent, location and histological type. Literature review suggests recurrence rates of around $8 \%$ in radical treatments, and $38 \%$ in conservative treatments. ${ }^{4}$ These figures are like those of a single center Australian study of 34 solid multicystic ameloblastomas treated by both conservative and aggressive techniques. ${ }^{15}$

In the present case, imaging findings, histopathological, and immunohistochemi-
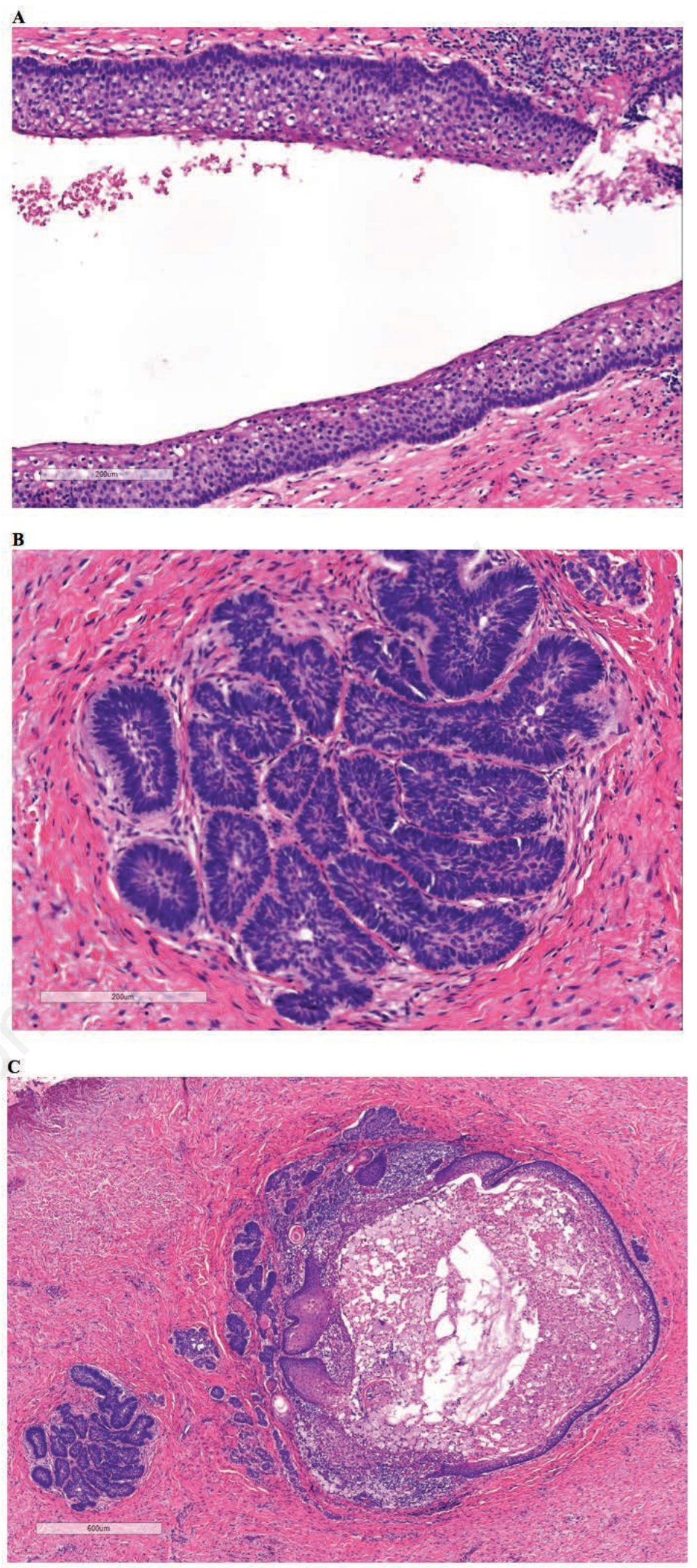

Figure 2. A) Histopathological features of odontogenic keratocyst (OKC). Hematoxylin and eosin showing a cyst wall with a thin layer of parakeratinised stratified squamous epithelium of six to eight cells in thickness and fibrous connective tissue, $x 200$ magnification. B) Histopathological features of ameloblastoma. Hematoxylin and eosin showing islands of odontogenic epithelium within a fibrous stroma, x200 magnification. C) Histopathological features of $\mathrm{OKC}$ and ameloblastoma, x100 magnification. 
cal assessment allowed for the reporting of definitive diagnosis of two pathological distinct entities: OKC and ameloblastoma. In the context of radiographic images, the $15 \mathrm{~mm}$ diameter unilocular expansile lowdensity lesion which diverges the intact roots of 42 and 43 is most likely to represent OKC (Figure 1A and B). Following surgical treatment, an OPG have shown medullary sclerosis at the site of removal of the lesion between 42 and 43 with distal angulation at the root of 43 (Figure 4). There is no evidence of recurrent radiolucency. It is very uncommon to detect the occurrence of two distinct odontogenic lesions simultaneously in one patient. The occurrence of ameloblastoma with orthokeratinized odontogenic cyst and glandular odontogenic cyst have been previously reported in 2006 and 2000, respectively. ${ }^{16,17}$ Gupta et al. reported a case of the simultaneous occurrence of unicystic ameloblastoma and $\mathrm{OKC}$ as completely distinct lesions in the posterior region of the mandible of a 22 years old male. ${ }^{18}$ Similarly, the synchronous occurrence of $\mathrm{OKC}$ and ameloblastoma in the mandible of a 45 years old male was reported. ${ }^{19}$ These two pathologically distinct entities were diagnosed following incisional and excisional biopsies ${ }^{19}$ (Table 1). Another reported case demonstrating simultaneous occurrences of odontogenic cysts and/or tumors include cemento-ossifying fibroma and $\mathrm{OKC}$ in the mandible, reported by Shimamoto et al. ${ }^{20}$

Whilst pre-surgical biopsy may have caught the diagnosis of ameloblastoma and changed the surgery performed, there is the possibility it may have been missed in the sample biopsied. If the diagnosis of ameloblastoma had been known pre-surgically, it would likely have led to the decision to extract the involved teeth with consideration of marginal mandibulectomy in the area. Following surgery and diagnosis, the decision was not to re-enter the site and continue with surveillance only.

The present case confirmed the possible synchronous occurrence of distinct histopathological entities in the mandible of a 57 years old female patient, OKC and ameloblastoma. This case also highlights the importance of identifying accurate diagnoses for such lesions which may prompt clinical implications. The potential high recurrence rates for both entities diagnosed in this case indicate the need for careful management plan and prevention of recurrence.

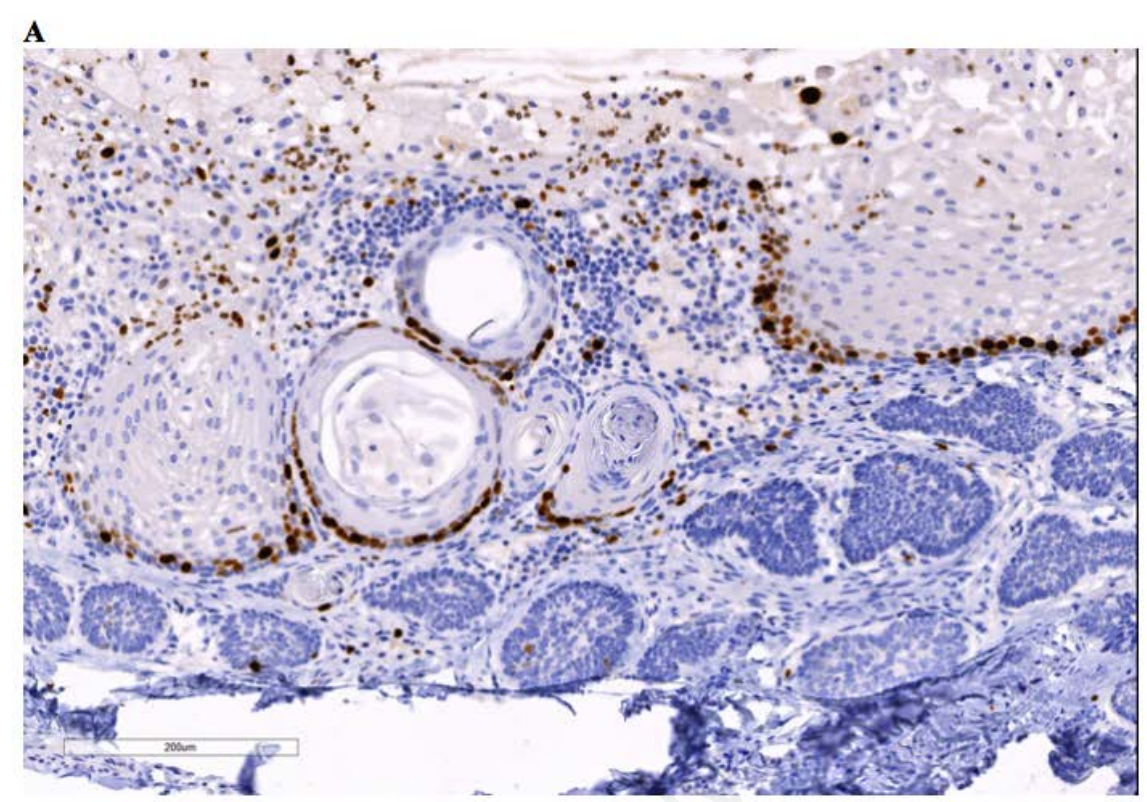

B

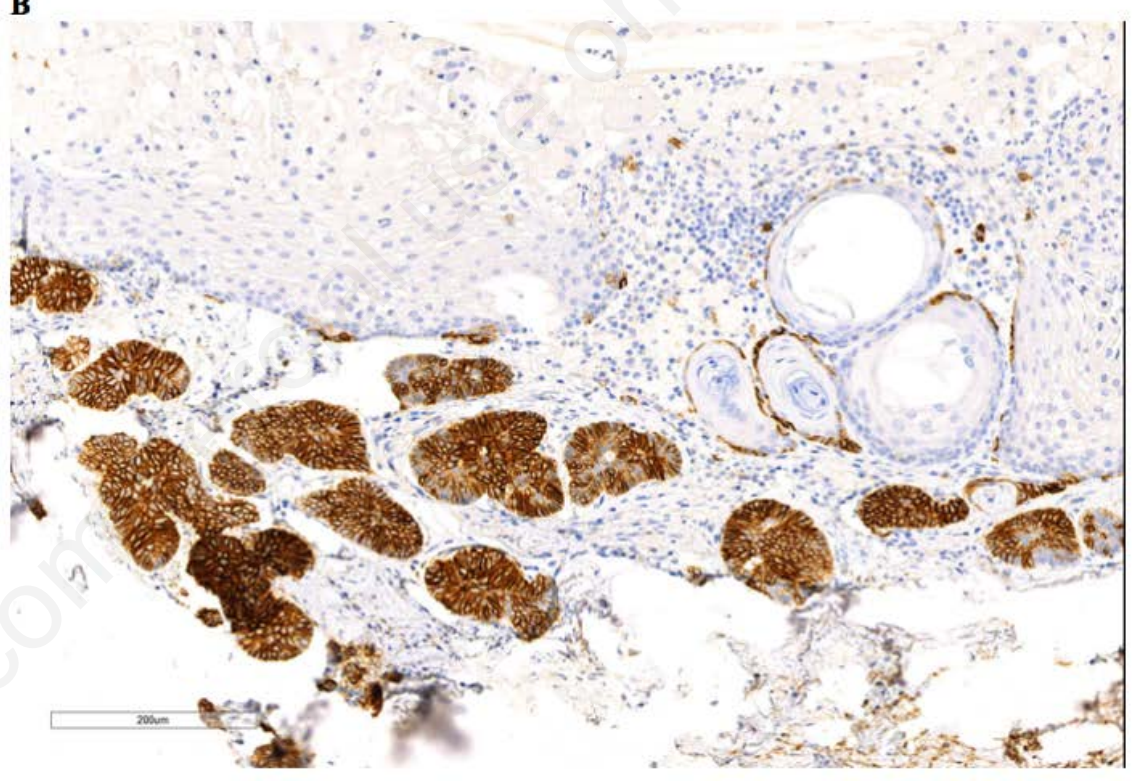

Figure 3. A) Immunohistochemical staining of Ki-67 in both ameloblastoma and odontogenic keratocyst (OKC), x200 magnification (note the high proliferation index in OKC). B) Immunohistochemical staining of CD56 (NCAM) in both ameloblastoma and $\mathrm{OKC}, \mathrm{x} 200$ magnification (note the strong positive staining in ameloblastoma islands and almost negative in $\mathrm{OKC})$.

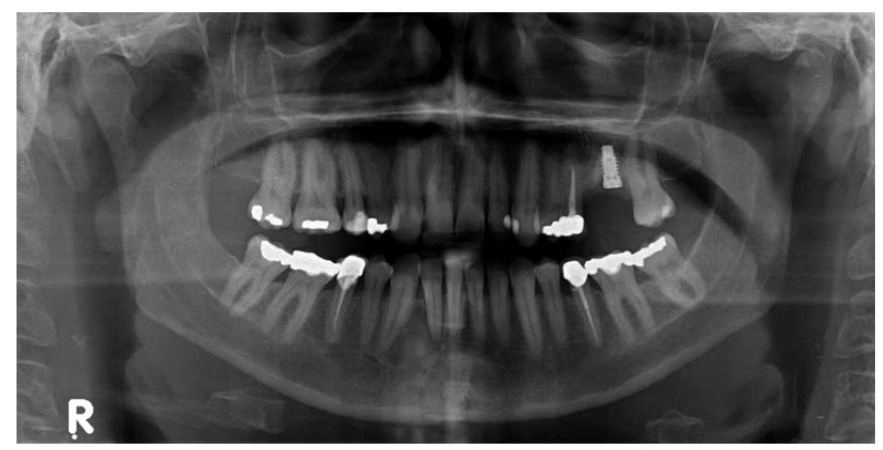

Figure 4. Orthopantomogram. Progressive osseous healing in the $42 / 43$ region with distal angulation at the root of 43 . There is no evidence of recurrent radiolucency. 
Table 1. Clinical, radiological, and histopathological features of case reports of synchronous ameloblastoma and odontogenic ameloblastoma.

$\begin{array}{cll}\text { Authors/date Age Gender Site } & \text { Recurrence/ Radiological Histopathological } \\ \text { (years) } & \text { follow-up duration } & \text { description }\end{array}$

\begin{tabular}{|c|c|c|c|c|c|}
\hline $\begin{array}{l}\text { Fergnani et al., } \\
2006^{16}\end{array}$ & 21 & Male & $\begin{array}{l}\text { Right angle and } \\
\text { of the mandible }\end{array}$ & $\begin{array}{l}\text { No/8 year } \\
\text { ascending ramus }\end{array}$ & $\begin{array}{l}\text { Large multilocular } \\
\text { radiolucency associated } \\
\text { with an impacted third molar }\end{array}$ \\
\hline
\end{tabular}

\begin{tabular}{|c|c|c|c|c|c|c|}
\hline $\begin{array}{l}\text { Gamoh et al., } \\
2015^{19}\end{array}$ & 45 & Male & $\begin{array}{l}\text { Right mandible } \\
\text { (premolar region) }\end{array}$ & No/6 month & $\begin{array}{l}\text { A 15-mm sized, round-style, } \\
\text { well-defined cystic } \\
\text { lesion on the root apex of } \\
\text { lower-right first molar; } \\
\text { in addition to a 12-mm sized, } \\
\text { radiolucent lesion in the } \\
\text { inter-alveolar septum of } \\
\text { the lower-premolars }\end{array}$ & $\begin{array}{l}\text { One cystic lesion lined by a thin layer of parak- } \\
\text { eratinized stratified squamous epithelium and } \\
\text { fibrous connective tissue. The other one has } \\
\text { odontogenic epithelium, structured stellate } \\
\text { reticulum and peripheral palisading } \\
\text { within a fibrous stroma }\end{array}$ \\
\hline $\begin{array}{l}\text { Gupta et al., } \\
2016^{18}\end{array}$ & 22 & Male & $\begin{array}{l}\text { Right mandible } \\
\text { (molar region) }\end{array}$ & Not available & $\begin{array}{l}\text { Round, well-defined cystic } \\
\text { lesion extending } \\
\text { from the root apex of the lower } \\
\text { left second molar causing root } \\
\text { resorption and extending } \\
\text { up to third molar }\end{array}$ & $\begin{array}{l}\text { Thin layer of regularly parakeratinized } \\
\text { stratified squamous epithelium and fibrous } \\
\text { connective tissue, in addition to a trabeculation } \\
\text { of enamel organ-like tissue with proliferation of } \\
\text { epithelium into stellate reticulum like cells }\end{array}$ \\
\hline Present case & 57 & Female & Anterior mandible & No/18 month & $\begin{array}{l}15 \mathrm{~mm} \text { unilocular lesion located } \\
\text { between the } 42 \text { and } 43 \text { roots }\end{array}$ & $\begin{array}{l}\text { Cyst with a thick fibrous connective tissue wall, } \\
\text { lined by parakeratinised stratified squamous } \\
\text { epithelium, in addition to islands of odontogenic } \\
\text { epithelium within a fibrous stroma in which the } \\
\text { cells are palisaded, columnar and hyperchromatic }\end{array}$ \\
\hline
\end{tabular}

\section{References}

1. El-Naggar A, Chan JK, Grandis JR, et al. World Health Organization classification of tumours: pathology and genetics: head and neck tumours. 4th edn. Lyon, France: IARC Press 2017.

2. Li TJ. The odontogenic keratocyst: a cyst, or a cystic neoplasm? J Dent Res 2011;90:133-42.

3. Guo YY, Zhang JY, Li XF, et al. PTCH1 gene mutations in Keratocystic odontogenic tumors: a study of 43 Chinese patients and a systematic review. PLoS One 2013;8:e77305.

4. Antonoglou GN, Sandor GK, Koidou VP, Papageorgiou SN. Non-syndromic and syndromic keratocystic odontogenic tumors: systematic review and metaanalysis of recurrences. J Craniomaxillofac Surg 2014;42:e364-71.

5. McClary AC, West RB, McClary AC, et al. Ameloblastoma: a clinical review and trends in management. Eur Arch Otorhinolaryngol 2016;273:1649-61.

6. Reichart PA, Philipsen HP, Sonner S. Ameloblastoma: biological profile of 3677 cases. Eur J Cancer B Oral Oncol 1995;31b:86-99.

7. Ariji Y, Morita M, Katsumata A, et al. Imaging features contributing to the diagnosis of ameloblastomas and keratocystic odontogenic tumours: logistic regression analysis. Dentomaxillofac Radiol
2011;40:133-40.

8. Heikinheimo K, Kurppa KJ, Laiho A, et al. Early dental epithelial transcription factors distinguish ameloblastoma from keratocystic odontogenic tumor. J Dent Res. 2015;94:101-11.

9. Becelli R, Carboni A, Cerulli G, et al. Mandibular ameloblastoma: analysis of surgical treatment carried out in 60 patients between 1977 and 1998. J Craniofac Surg 2002;13:395-400; discussion 00.

10. Schafer DR, Thompson LD, Smith BC, Wenig BM. Primary ameloblastoma of the sinonasal tract: a clinicopathologic study of 24 cases. Cancer 1998;82:66774.

11. Fujita M, Matsuzaki H, Yanagi Y, et al. Diagnostic value of MRI for odontogenic tumours. Dentomaxillofac Radiol 2013;42:20120265.

12. Hammarfjord O, Roslund J, Abrahamsson P, et al. Surgical treatment of recurring ameloblastoma, are there options? Br J Oral Maxillofac Surg 2013;51:762-6.

13. Becelli R, Morello R, Renzi G, et al. Treatment of recurrent mandibular ameloblastoma with segmental resection and revascularized fibula free flap. J Craniofac Surg 2011;22:1163-5.

14. Carlson ER, Marx RE. The ameloblastoma: primary, curative surgical management. J Oral Maxillofac Surg 2006;64:
Cystic cavity lined by squamous and stratified epithelium with a thick layer of orthokeratin, in addition to an area of plexiforme ameloblastoma odontogenic epithelium, structured stellate reticulum and peripheral palisading within a fibrous stroma

Thin layer of regularly parakeratinized stratified squamous epithelium and fibrous connective tissue, in addition to a trabeculation of enamel organ-like tissue with proliferation of epithelium into stellate reticulum like cells

Cyst with a thick fibrous connective tissue wall, lined by parakeratinised stratified squamous epithelium within a fibrous stroma in which the cells are palisaded, columnar and hyperchromatic

484-94.

15. Singh T, Wiesenfeld D, Clement J, et al. Ameloblastoma: demographic data and treatment outcomes from Melbourne, Australia. Aust Dent J 2015;60:24-9.

16. Fregnani ER, da Cruz Perez DE, Soares FA, Alves FA. Synchronous ameloblastoma and orthokeratinized odontogenic cyst of the mandible. J Oral Pathol Med 2006;35:573-5.

17. Hisatomi M, Asaumi J, Konouchi H, et al. A case of glandular odontogenic cyst associated with ameloblastoma: correlation of diagnostic imaging with histopathological features. Dentomaxillofac Radiol 2000;29:249-53.

18. Gupta RK, Dugal AG, Pawar SR, et al. A rare simultaneous occurrence of odontogenic keratocyst and unicystic ameloblastoma in mandible: a case report. J Clin Diagn Res 2016;10:Zd01-4.

19. Gamoh S, Akiyama H, Tominaga K, et al. Simultaneous occurrence of keratocystic odontogenic tumor and ameloblastoma in the mandible: A case report. Oncol Lett 2015;10:785-89.

20. Shimamoto H, Kishino M, Okura M, et al. Radiographic features of a patient with both cemento-ossifying fibroma and keratocystic odontogenic tumor in the mandible: a case report and review of literature. Oral Surg Oral Med Oral Pathol Oral Radiol Endod 2011;112:798-802. 